\title{
Effect of distraction task on driving performance of experienced taxi drivers
}

\author{
Sang-Hyeok Seo', Seung-Hyun Kwak', Soon-Cheol Chung ${ }^{2}$, Hyung-Sik Kim 2,*,\#, \\ Byung-Chan Min ${ }^{1, *}$
}

Abstract

Background: Driving performance is influenced by human, vehicular, and environmental factors.

Objectives: To investigate the effects of distraction tasks, such as sending a text message (STM) and searching a navigation device (SN), on the driving performance of experienced taxi drivers.

Methods: Twelve male taxi drivers (age: $56.3 \pm 4.4 \mathrm{y}$; experience: $28.4 \pm 6.4 \mathrm{y}$ ) and 14 female taxi drivers (age: $55.5 \pm 3.5 \mathrm{y}$; experience: $19.4 \pm 5.0 \mathrm{y})$ drove in a simulator at a constant speed $(90 \mathrm{~km} / \mathrm{h})$ for 2 min while maintaining a gap of $30 \mathrm{~m}$ from the car in front, also traveling at $90 \mathrm{~km} / \mathrm{h}$. Participants were instructed to drive only for the first $1 \mathrm{~min}$ (control phase). For an additional $1 \mathrm{~min}$ (task phase), they were instructed to drive only, drive $+\mathrm{STM}$, or drive $+\mathrm{SN}$. Results: Compared with driving only, during driving + STM or driving + SN, the drivers' skin conductance level was relatively increased, suggesting that the distraction task increased the drivers' workload and sympathetic nervous system activity. Compared with driving only, during driving + STM or driving + SN, the average distance from the car in front, speed deviation, and anterior-posterior and medial-lateral coefficients of variation increased, suggesting that maintaining the instructed gap and speed, and the longitudinal and transverse control of the car, was more difficult because of the distraction task.

Conclusions: Even for highly experienced taxi drivers, distraction tasks increased workload, increased the difficulty of vehicle control, and detracted from safe driving.

Keywords: distraction task, driving performance, experienced drivers, sympathetic nervous system, workload

The 21 st century has seen a rise in the vehicular use of electronic equipment, such as mobile phones and navigation systems. Mobile phones are useful communication tools and sometimes important in an emergency situation. Navigation systems often provide not only path-finding information but also music, movie, and digital multimedia broadcasting services. Navigation systems also increase driving convenience by providing real-time road and traffic information. While the use of in-vehicle electronics can increase the quality of the driving experience, it is conversely possible that the additional

*Correspondence to: Hyung-Sik Kim, Department of Biomedical Engineering, Research Institute of Biomedical Engineering, College of Science and Technology, Konkuk University, 268 Chungwon-daero, Chungju-si, Chungbuk 380-701, South Korea, e-mail: hskim98@kku.ac.kr; "These authors contributed equally to this work.

'Department of Industrial and Management Engineering, Hanbat National University, Daejeon 305-719, South Korea

${ }^{2}$ Department of Biomedical Engineering, Research Institute of Biomedical Engineering, College of Science and Technology, Konkuk University, Chungju-si 380-701, South Korea

(c) (1) (-) () 2017 Sang-Hyeok Seo, Seung-Hyun Kwak, Soon-Cheol Chung, Hyung-Sik Kim, Byung-Chan Min

This Work is licensed under Creative Common License 
information can increase the workload of drivers and decrease their attention, which could result in serious problems such as increased risk of accidents [1-3].

According to the United States National Highway Traffic Safety Administration, during 2008, distracted attention during driving led to the death of 5,870 drivers and injury of 500,000 drivers in the USA [4]. Distraction of the driver is considered responsible for $25 \%-80 \%$ of vehicle-related accidents [5]. Performing secondary tasks while driving increases the possibility of a car crash [6].

Numerous studies have been undertaken to evaluate the effects of performing a task or disruption of attention on driving. The use of a mobile phone or navigation system during driving increases average following distance, decreases average car speed, and increases variability of car speed [7-13]. Use of the electronic devices also increases the anterior-posterior coefficient of variation (APCV) and medial-lateral coefficient of variation (MLCV) of the car [7, 8, 14]. Furthermore, the use of a mobile phone or navigation system increases driver workload and influences the stress experienced by the driver. Such distractions activate the sympathetic nervous system and increase the heart rate and skin conductance level (SCL) of drivers [15-19].

Driving performance is influenced by a combination of human, vehicular, and environmental factors. Because of a flaw in one or more of these factors, accidents occur wherein the main cause is most often the human factor [20]. Accidents because of human factors occur when the driver is inexperienced or careless (e.g., fails to respond to traffic signals and warnings) [21, 22]. Driving is a complicated activity requiring focused attention by the driver; secondary tasks performed while driving can impair driving ability [23]. Driver distraction because of additional workload can trigger mistakes [7-19]. However, these previous studies have been mostly performed using young adults with relatively short driving experience, and few studies of experienced drivers with extensive driving experience have been conducted. Because driving performance is influenced by driving experience, further knowledge is needed to clarify the effects of distraction tasks on the performance of experienced drivers.

This study was undertaken to investigate the effects of distraction on driving performance of experienced taxi drivers in their 50s. Distraction tasks used in earlier studies, such as sending a text message (STM) and searching a navigation device (SN), were selected in this study. Car control parameters including average following distance, speed deviation, APCV, and MLCV were used to evaluate driving performance. Change in activation level of the sympathetic nervous system was investigated using SCL.

\section{Methods}

\section{Driving simulator}

The GDS-300s driving simulator (Gridspace Inc., Korea) provides front, left, and right visual information via 3 32-inch liquid crystal display monitors. The driving simulator is similar to the 'Click' model from Hyundai Motors, having driving devices (steering wheel, accelerating pedal, brake pedal, parking brake, direction indicator lever, emergency lamp lever, windshield wiper lever, headlight lever, gear lever, and safety belt lamp) and displays (direction indicator lamp, speedometer, revolutions per minute meter, temperature gauge, fuel gauge, and several warning lights). Motor-driven power steering, which is a motor control type simulator, was used as the steering wheel.

\section{Participants}

The protocol for the research project was approved by the Institutional Review Committee of Konkuk University (KUH116062) where the work was undertaken, and the protocol conforms to the principles of the Declaration of Helsinki and its contemporary amendments. The overall purpose and protocol for the experiments were explained to the participants before their enrollment in the study, and experiments were conducted after written consent informed was obtained.

Twelve male taxi drivers (age: $56.3 \pm 4.4 \mathrm{y}$, with $28.4 \pm$ 6.4 y taxi-driving experience) and 14 female taxi drivers (age: $55.5 \pm 3.5 \mathrm{y}$, with $19.4 \pm 5.0 \mathrm{y}$ taxi-driving experience) participated in this study. Before starting the experiment, each participant was familiarized with the driving simulator through a practice driving session. The experimental design was controlled for external factors, such as the use of tobacco, alcohol, or coffee, which could possibly influence driving performance and physiological signals.

\section{Experimental procedure}

Participants were instructed to drive without changing lanes, while maintaining a constant $30 \mathrm{~m}$ headway gap from the car ahead on a 3-lane straight road in a vacant downtown area. The front car maintained a speed of $90 \mathrm{~km} / \mathrm{h}$. The headway distance was displayed on the screen of the simulator in real time. The experimental paradigm is shown in Figure 1.

After resting in the driver's seat for 3 min (rest phase), participants drove for 2 min while maintaining a constant gap 
(30 m) and speed $(90 \mathrm{~km} / \mathrm{h})$. Participants were driving only for the first $1 \mathrm{~min}$ (control phase). For an additional $1 \mathrm{~min}$, they were instructed to drive only or perform a task (STM or SN) while driving (task phase). For a task that involves following the car in front, if the task takes too long, the driver may feel bored and may not be able to focus on the experiment. Therefore, as suggested by preliminary experiments, the driving time was restricted to 2 min so that most subjects did not feel bored. In the case of STM, subjects were instructed to use their own mobile phones and put the phone in the most convenient place they could find within $30 \mathrm{~cm}$ of the steering wheel (Figure 2).

In the case of $\mathrm{SN}$, participants were instructed to use the navigation system provided by the experimenter who placed a car-mounted-type navigation on the dashboard. In particular, when participants were not accustomed to the navigation, they were instructed to familiarize themselves with and practice use of the navigation system in advance. All subjects were right handed and they were requested to conduct the tasks with their right hand. All subjects participated in 3 types of experiments: driving only, driving + STM, and driving + SN. During the experiments, the experimenter counted the number of crashes with the car in front. The order of experiments was counterbalanced. In detail, after completing one experiment, participants

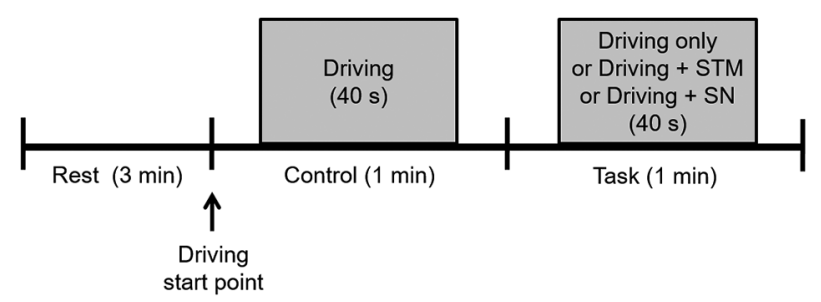

Figure 1. Experimental paradigm

STM, sending a text message; $\mathrm{SN}$, searching a navigation device took a 30-min rest to account for possible simulator sickness before moving on to the next experiment. The experiment time was approximately $1.5 \mathrm{~h}$ for each participant.

\section{SCL measurements}

The activation level of the sympathetic nervous system was measured using SCL. An MP100 data acquisition system and AcqKnowledge version 3.8.1 (Biopac Systems) were used to measure the SCL from the index and middle fingers on the left hand. The sampling rate of the physiological data was 500 samples/s.

\section{Data analysis}

The relative change of SCL was calculated, which was obtained by dividing the difference between the SCL in the task phase $\left(\mathrm{SCL}_{\text {Task }}\right)$ and the $\mathrm{SCL}$ in the control phase $\left(\mathrm{SCL}_{\mathrm{Control}}\right)$ by the $\mathrm{SCL}_{\text {Control }}$ (Eq. 1).

$$
\begin{aligned}
\text { Relative change of SCL }(\%)= & \frac{\mathrm{SCL}_{\text {Task }}-\mathrm{SCL}_{\text {Control }}}{\mathrm{SCL}_{\text {Control }}} \\
& \times 100
\end{aligned}
$$

The driving simulator supplied information on the distance from the car ahead as well as the vehicle's speed and location. Using the information regarding the distance from the car ahead, the average following distance was calculated.

The speed deviation was calculated by using the vehicle speed and obtained by dividing the difference between the intended design speed $(90 \mathrm{~km} / \mathrm{h})$ and operating speed by the design speed (Eq. 2).

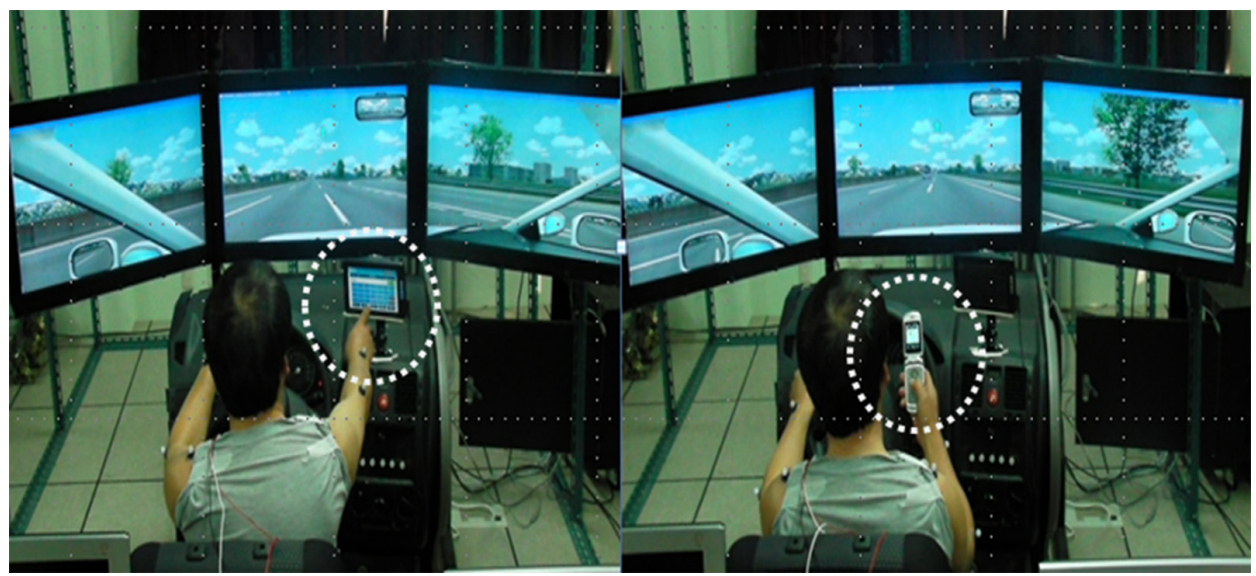

Figure 2. Task performance while driving 


$$
\begin{aligned}
\text { Speed deviation }(\%)= & \frac{\text { Design speed }- \text { Operating speed }}{\text { Design speed }} \\
& \times 100
\end{aligned}
$$

Using the information on distance from the car ahead, the APCV, which represents vertical control of the car, was calculated. The APCV is obtained by dividing the standard deviation $\left(\mathrm{AP}_{\mathrm{STD}}\right)$ of the distance from the car ahead by the mean value $\left(\mathrm{AP}_{\text {Mean }}\right)$ (Eq. 3). The $\mathrm{APCV}$, as an indicator showing the variability of the front and rear movements of a car, is one of the control variables influenced by the driving performance.

$$
\mathrm{APCV}=\frac{\mathrm{AP}_{\mathrm{STD}}}{\mathrm{AP}_{\mathrm{Mean}}}
$$

Using the location data of the car from the driving simulator, the MLCV, which describes the lateral controllability of the car, was calculated. MLCV was defined by Eq. 4, the standard deviation of the positional coordinates of the mediallateral movement $\left(\mathrm{ML}_{\mathrm{STD}}\right)$ of the car divided by the mean value $\left(\mathrm{ML}_{\text {Mean }}\right)$. This is an index explaining the variation in the left and right car movements in the center of the driving lane and is one of several control variables influenced by driving performance.

$$
\mathrm{MLCV}=\frac{\mathrm{ML}_{\mathrm{STD}}}{\mathrm{ML}_{\text {Mean }}}
$$

Relative change of SCL was calculated for $40 \mathrm{~s}$, excluding the first $10 \mathrm{~s}$ and final $10 \mathrm{~s}$ in the control phase and the task phase that included driving only, driving + STM, and driving + $\mathrm{SN}$. Average following distance, speed deviation, APCV, and MLCV were calculated for $40 \mathrm{~s}$, excluding the first $10 \mathrm{~s}$ and final $10 \mathrm{~s}$ in the task phase including driving only, driving + STM, and driving + SN (Figure 1).

Concerning the factors relative change of SCL, average following distance, speed deviation, APCV, and MLCV, a repeated one-way analysis of variance, which considers an independent variable condition (driving only, driving + STM, and driving $+\mathrm{SN}$ ) was conducted (SPSS, version 12.0k).

\section{Results}

\section{Relative change of SCL}

As seen from Table 1, significant differences were found in the relative change of SCL between driving only, driving + STM, and driving $+\mathrm{SN}(F=11.8, P<0.001)$. Bonferroni post hoc tests showed (Figure 3) that the relative change of SCL of driving $+\mathrm{SN}(P<0.001)$ and driving $+\mathrm{STM}(P<0.01)$ increased significantly, compared with that of driving only.

\section{Average following distance}

As seen from Table 1, there were significant differences in the average following distance between driving only, driving + $\mathrm{STM}$, and driving $+\mathrm{SN}(F=28.4, P<0.001)$. Bonferroni post hoc tests showed (Figure 4) that the average following distance of driving $+\mathrm{SN}$ increased significantly, compared with that of driving only $(P<0.001)$ and compared with that of driving + STM $(P<0.05)$. The average following distance of driving + STM increased significantly, compared with that of driving only $(P<0.001)$

Table 1. Mean and standard deviation according to conditions (driving only, driving + sending text message [STM], driving + searching a navigation device [SN]) in the relative change of skin conductance level (SCL), average following distance, speed deviation, anterior-posterior coefficient of variation (APCV), and medial-lateral coefficient of variation (MLCV)

\begin{tabular}{lrrr}
\hline Factors & Driving only & Driving + STM & Driving + SN \\
\hline $\begin{array}{l}\text { Relative change of } \\
\text { SCL, \% }\end{array}$ & $0.13 \pm 1.98$ & $4.79 \pm 5.88$ & $6.15 \pm 6.46$ \\
Average following & $48.17 \pm 21.23$ & $99.33 \pm 51.31$ & $127.79 \pm 70.99$ \\
distance, $m$ & & & \\
Speed deviation, \% & $4.33 \pm 1.84$ & $12.54 \pm 8.35$ & $16.88 \pm 9.47$ \\
APCV & $0.17 \pm 0.09$ & $0.40 \pm 0.27$ & $0.50 \pm 0.33$ \\
MLCV & $0.11 \pm 0.02$ & $0.24 \pm 0.06$ & $0.35 \pm 0.16$ \\
\hline
\end{tabular}

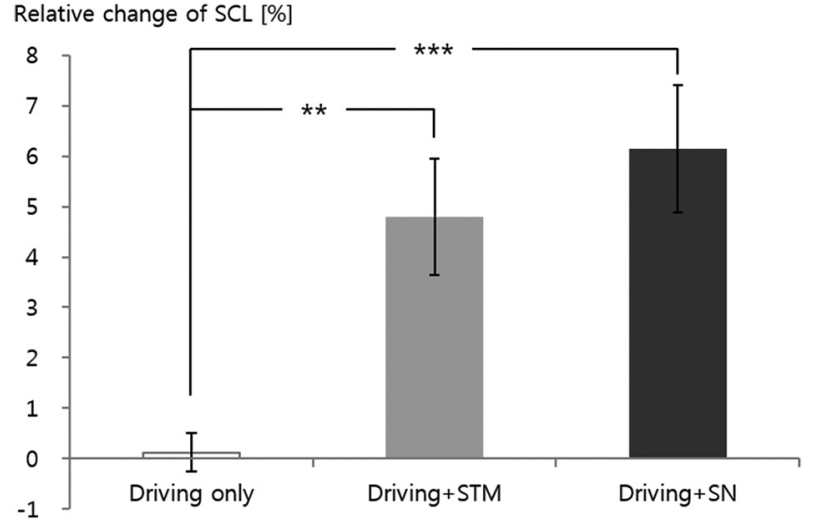

Figure 3. Relative change of skin conductance level $(\mathrm{SCL})$ during the 3 types of experiments

***P $P<0.001,{ }^{* *} P<0.01$

STM, sending a text message; $\mathrm{SN}$, searching a navigation device 


\section{Speed deviation}

As seen from Table 1, significant differences were found in the speed deviation between driving only, driving + STM, and driving $+\mathrm{SN}(F=25.5, P<0.001)$. Bonferroni post hoc tests showed (Figure 5) that the speed deviation of driving $+\mathrm{SN}$ $(P<0.001)$ and driving + STM $(P<0.001)$ increased significantly, compared with that of driving only.

\section{Anterior-posterior coefficient of variation observations}

As seen from Table 1, there were significant differences found in the APCV between driving only, driving + STM, and driving $+\mathrm{SN}(F=16.7, P<0.001)$. Bonferroni post hoc tests

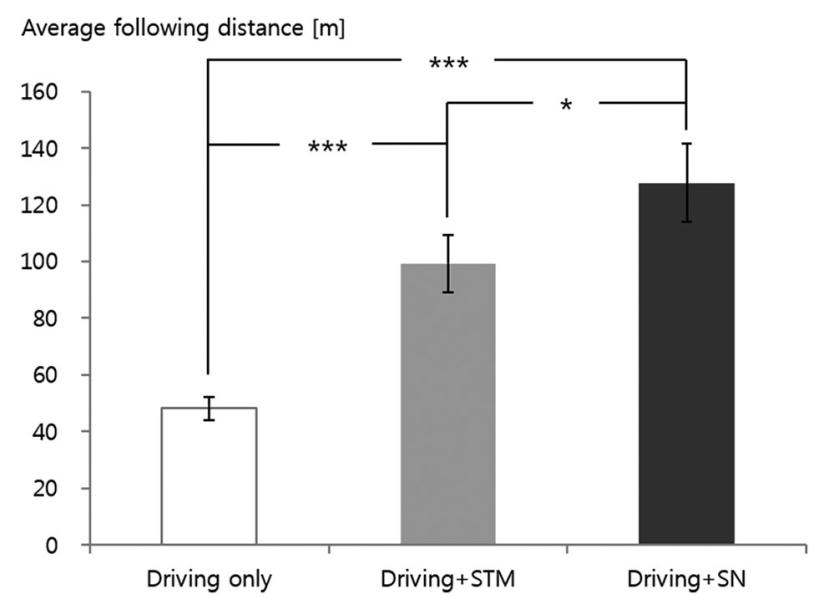

Figure 4. Average following distance during the 3 types of experiments *** $P<0.001, * P<0.05$

STM, sending a text message; SN, searching a navigation device

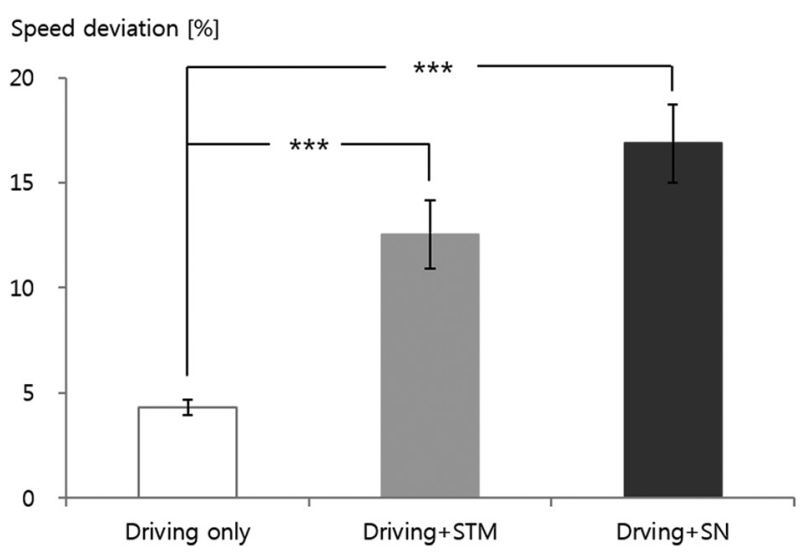

Figure 5. Speed deviation in the 3 types of experiments *** $P<0.001$

STM, sending a text message; $\mathrm{SN}$, searching a navigation device showed (Figure 6) that the APCV of driving $+\mathrm{SN}$ increased significantly compared to that of driving only $(P<0.001)$ and that of driving $+\mathrm{STM}(P<0.05)$. The APCV of driving + STM increased significantly compared with that of driving only $(P<0.01)$. There were no crashes with the car in front during the experiments.

\section{Medial-lateral coefficient of variation observations}

As seen from Table 1, significant differences were found in the MLCV between driving only, driving + STM, and driving + SN $(F=42.4, P<0.001)$. Bonferroni post hoc tests showed (Figure 7) that the MLCV of driving $+\mathrm{SN}$ increased significantly, compared

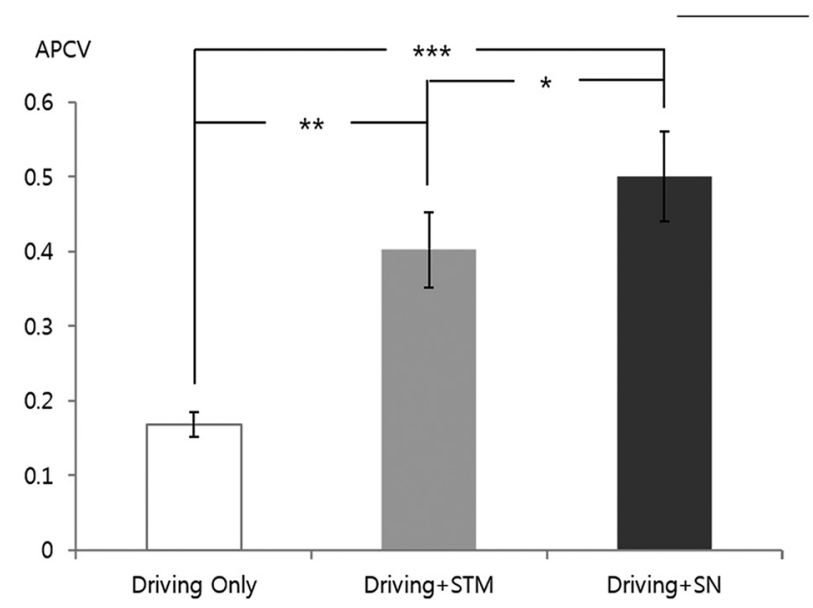

Figure 6. Anterior-posterior coefficient of variation (APCV) for the 3 types of experiments *** $P<0.001,{ }^{* *} P<0.01, * P<0.05$

STM, sending a text message; $\mathrm{SN}$, searching a navigation device

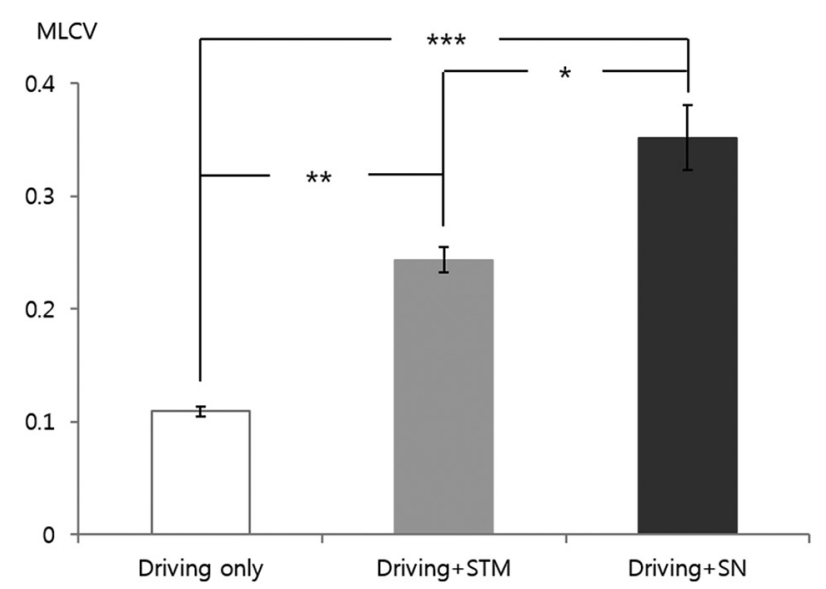

Figure 7. Medial-lateral coefficient of variation (MLCV) for the 3 types of experiments

*** $P<0.001,{ }^{* *} P<0.01,{ }^{*} P<0.05$

STM, sending a text message; $\mathrm{SN}$, searching a navigation device 
with that of driving only $(P<0.001)$ and that of driving + STM $(P<0.05)$. The MLCV of driving + STM increased significantly compared with that of driving only $(P<0.01)$.

\section{Discussion}

This study investigated the effects of distraction tasks such as STM and SN on the driving performance of experienced male and female taxi drivers more than $50 \mathrm{y}$ of age.

Compared with driving only, additional tasks increased the relative change in SCL significantly. This means that distraction tasks increased the workload of drivers and then increased the activity of the sympathetic nervous system. Increase in workload resulted in decreased driving performance. Because of the distraction tasks, increases in average following distance and speed deviation made it difficult to maintain the instructed following distance and speed. Increases in APCV and MLCV made it difficult to control the car longitudinally and transversely. We conclude that, even for experienced taxi drivers, distraction tasks increased driver workload and made it more difficult to control the car than when undistracted. The results of the present study are consistent with those of young adults with little driving experience [7-19]. If the participant is completely distracted during driving, collision with the vehicle in front may occur [24]. In the present experiment, no crash occurred. This is because the purpose of this study was to investigate the changes in driving performance caused by a distraction task, so the task was not designed to overload the participant to a level that is completely distracted.

Compared with STM, SN increased the relative change of SCL and decreased driving ability. It is relatively easy to manipulate a mobile phone for the STM task during driving than to conduct SN. However, for SN, the additional workload resulting from increased movement made it difficult to control the car, because the navigation system was placed in the central upper part of the dashboard. The loss of vehicular control may also result from the unfamiliarity of participants with the provided navigation system, compared with their own mobile phones. Complementary research on this topic will be necessary to determine differences.

Distraction tasks such as STM and SN during driving decrease driving performance of experienced taxi drivers in their 50s and made their driving less safe. In the experiment, it was assumed that a secondary task for a taxi driver who has a high automaticity of driving skill would not change the driving performance even though secondary experience was given. However, even for these participants, we confirmed that the task, by causing divided attention, distracted from driving.

Because driving performance can be influenced by driving experience and age, additional studies with these factors are necessary to investigate the effects of distraction tasks on driving performance more precisely. Even though the results of the present study are similar to those of earlier studies, direct comparison is unreasonable. It is unreasonable to compare quantitatively the results of young adults with little driving experience with those of older adults with extensive driving experience. Instead, more studies that control for the effects of driving experience, comparative study of groups of with different ages and the same driving experience, and studies that control for age-related effects by comparing various levels of driving experience within the same age group are needed to reveal the effects of distraction tasks on driving performance.

Limitations of the present study are as follows. The experiment in the present study used a real car, but the display of the driving environment was simulated to provide the same experimental conditions to each participant. The length of the experiments was short to avoid habituation to the experiments and boredom of the participants, which might have affected the results. These conditions may differ from real-world driving conditions. Comparative experiments under more realistic environmental conditions may be required to overcome these limitations.

\section{Conclusions}

Distraction tasks changed the drivers' sympathetic nervous system, increasing their level of skin conductance. Even for highly experienced taxi drivers, distraction tasks increased workload as seen by SCL, increased the difficulty of vehicle control, and detracted from safe driving.

Author contributions. SHS, HSK, and BCM conceived and designed the experiments. SHS and SHK recruited participants, conducted experiments, and acquired the data. SHS, SHK, and SCC analyzed and interpreted the data. SHS, SHK, SCC, HSK, and BCM drafted the article or revised it critically for important intellectual content. All authors approved the version submitted for publication and take full responsibility for all statements made in this article.

Acknowledgments. We especially thank Dr. Mi-Hyun Choi and Mr. Woo-Ram Kim for their efforts to set up the experimental environment and make comments on the manuscript. The authors thank all participants and staff for their assistance and help with the experiments.

This work was supported by a Mid-Career Researcher Program Grant through the National Research Foundation of Korea (NRF), funded by the Ministry of Education (MOE) (number 2017R1A2B2004629). 
Conflict of interest statement. The authors have no directly competing interests to disclose. The authors are listed as inventors in the registered Korean patent number 10-1345310, which describes laser-based technology for the realization of touch (or tactile sensation) as applied to the driving environment of skilled taxi drivers and daily drivers with secondary tasks, and they declare that they have no conflicts of interest.

\section{References}

[1] Anttila V, Luoma J. Surrogate in-vehicle information system and driver behaviour in an urban environment: a field study on the effects of visual and cognitive load. Transp Res Part F Traffic Psychol Behav. 2005; 8:121-33.

[2] Lee YT, Kim MH, Son JW. Effects of advancing age on driver's cognitive workload. Ergon Soc Korea. 2009; 28:73-9.

[3] Lee WS, Park JW, Kim SJ, Yoon SH, Yang X, Lee YT, et al. Development of an analysis system for biosignal and driving performance measurements. Ergon Soc Korea. 2010; 29:47-53.

[4] Ascone D, Lindsey T, Varghese C. An examination of driver distraction as recorded in NHTSA databases. National Highway Traffic Safety Administration DOT [on line]. 2009 Sep. (No. HS-811 216), Available from: http://www-nrd.nhtsa.dot.gov/Pubs/811216.pdf. Accessed January 2017.

[5] Dingus TA, Klauer SG, Neale VL, Petersen A, Lee SE, Sudweeks JD, et al. The 100-car naturalistic driving study: phase II-Results of the 100-car field experiment. National Highway Traffic Safety Administration DOT [on line]. 2006 Apr. (No. HS-810 593) Available from: http://www.nhtsa.gov/DOT/NHTSA/NRD/Multimedia/ PDFs/Crash\%20Avoidance/Driver\%20Distraction/100CarMain.pdf. Accessed January 2017.

[6] Neale VL, Dingus TA, Klauer SG, Sudweeks J, Goodman M. An overview of the 100-car naturalistic study and findings (05-0400). Proceedings of the 19th International Technical Conference on the Enhanced Safety of Vehicles; 2005 June 6-9, 2005; Washington DC, USA. Available from: http://www.dot.alaska.gov/highwaysafety/ assets/Occ_Prot-cellphone_National_study-Virginia_Tech_Transp_ Institute.pdf.

[7] Brumby DP, Salvucci DD, Howes A. Focus on driving: how cognitive constraints shape the adaptation of strategy when dialing while driving. Proceedings of the SIGCHI Conference on Human Factors in Computing Systems; 2009 April 4-9; Boston, USA. pp. 1629-38.

[8] Choi SH, Lee JS. The effects of cellular-phone use on driving performance under various driving speed conditions. Korean J Sci Emotion Sensibility. 2006; 6:1-11.

[9] Haigney DE, Taylor RG, Westerman SJ. Concurrent mobile (cellular) phone use and driving performance: task demand characteristics and compensatory processes. Transp Res Part F Traffic Psychol Behav. 2000; 3:113-21.
[10] Lee SW. Differential effects of in-vehicle electronic device usage types on driver's attentional distraction and driving performance. MSc thesis, Pusan National University, Pusan, Korea, 2008.

[11] Shin YK, Lim PN, Kang SC. The effects of in vehicle watching TV on driver behavior. Korean Soc Transport. 2006; 24:103-12.

[12] Shin YK, Ryu JB. The effect of operating telematics device in vehicle on driver behaviors. Korean Soc Transport. 2008; 26:39-47.

[13] Strayer DL, Drews FA, Crouch DJ, Johnson WA. Why do cell phone conversations interfere with driving? In: Walker WR, Herrmann DJ, editors. Cognitive technology: essays on the transformation of thought and society. Jefferson, NC, USA: McFarland and Company; 2005, p. 51-68.

[14] Mun KR, Choi JS, Kang DW, Bang YH, Kim HS, Lee SJ, et al. The effects of secondary task on driving performance: control of vehicle and analysis of motion signal. Korean J Sci Emotion Sensibility. 2010; 13:613-20.

[15] Choi YI, Park KS, Eum KS. The Effects of concentration level of telephone conversation on the automobile driver's performance. KSCE Civil Eng. 2007; 27:165-69.

[16] Collet C, Clarion A, Morel M, Chapon A, Petit C. Physiological and behavioural changes associated to the management of secondary tasks while driving. Appl Ergon. 2009; 40:1041-46.

[17] Kim YS. A study on driver distraction using a driving simulator. MSc, Kookmin University, Seoul, Korea, 2010.

[18] Koo TY, Kim BY, Ji SH, Suh MW. A study on workload of using telematics while driving. Trans Korean Soc Automot Eng. 2009; 17:26-33.

[19] Yang JW, Lee SJ, Kim JH, Choi MH, Choi JS, Kim HS, et al. Effects of sending text message and searching navigation on skin conductance level and deviation of vehicle speed during driving. J Soc Korea Ind Syst Eng. 2011; 34:9-14.

[20] Wester AE, Bocker KB, Volkerts ER, Verster JC, Kenemans JL. Event-related potentials and secondary task performance during simulated driving. Accid Anal Prev. 2008; 40:1-7.

[21] Merat N, Anttila V, Luoma J. Comparing the driving performance of average and older drivers: the effect of surrogate in-vehicle information systems. Transp Res Part F Traffic Psychol Behav. 2005; 8:147-66.

[22] Summala H. Accident risk and driver behavior. Saf Sci. 1996; 22:103-17.

[23] Bellet T, Bailly-Asuni B, Mayenobe P, Banet A. A theoretical and methodological framework for studying and modelling drivers' mental representations. Saf Sci. 2009; 47:1205-21.

[24] Hickman JS, Hanowski RJ, Bocanegra J. Distraction in commercial trucks and buses: assessing prevalence and risk in conjunction with crashes and near-crashes. Report No. FMCSA-RRR-10-049. Washington, DC: U.S. Department of Transportation. Federal Motor Carrier Safety Administration; 2010 Sept. Available from: http://citeseerx.ist.psu.edu/viewdoc/ summary?doi=10.1.1.173.3995. 
